\title{
An Open-Label Observational Trial to Evaluate the Possible Effects of Individualized Homoeopathic Medicines in Symptomatic Nasal Polyp
}

\author{
Aniruddha Banerjee, ${ }^{1}$ Birendra Prasad Srivastava, ${ }^{2}$ Munmun Koley, ${ }^{3}$ Subhranil Saha ${ }^{3}$
}

\begin{abstract}
Introduction
Nasal polyps presenting with chronic rhino-sinusitis (CRS) is a commonly encountered condition characterized by nasal obstruction, loss of sense of smell (anosmia), postnasal drip, headache, and sleep disorders. Possibly 60-65\% of the populations suffering from CRS has predisposition to nasal polyps. Homoeopathic literature claims to offer successful treatment of nasal polyps; but scarcely subjected to systematic research.

Materials and Methods

A prospective, open, non-randomized, single arm, observational trial of pre-post comparison design was conducted on 44 patients suffering from symptomatic nasal polyps. Sino-nasal outcome test (SNOT-20) and European Quality of Life (EQ-5D$5 L)$ questionnaires were taken as the primary and secondary outcome measures respectively; assessed at baseline and after 3 months. Individualized homoeopathic medicines were prescribed on 'totality of symptoms'. Intention to treat sample was subjected to statistical analysis. Data distribution was examined. Non-parametric Wilcoxon signed rank test and post hoc parametric paired t test were used accordingly. P values less than 0.05 were considered as statistically significant.

$\underline{\text { Results }}$

Forty four patients were enrolled; 4 dropped out. Skiagrams revealed complete regressions of polyps in 23 (58\%) cases. Statistically significant improvements were observed in both the subjective patient-rated outcomes - mean reduction of SNOT20 scores by 19.9 [sd 15.5; 95\% CI 15.5 to 24.2; P < 0.001], EQ-5D-5L questionnaire score by 0.9 [sd 2.6; $95 \%$ CI 0.1 to 1.7; $P=0.029]$; and $E Q-5 D-5 L$ VAS by 14.4 [sd 4.7; $95 \%$ CI 12.9 to 15.9; $P<0.001]$.

Conclusion

Homoeopathic medicines showed promising treatment effect in symptomatic nasal polyps. Randomized trials are warranted..

$\underline{\text { Kevwords }}$

Homeopathy; Nasal Polyp; Quality of Life
\end{abstract}

$\underline{\text { ABSTRACT }}$

$\mathrm{N}$ asal polyps (NP) presenting with the symptoms of chronic rhino-sinusitis (CRS) is one commonly prevalent disorders of the nose and para-nasal sinuses. These are non-cancerous growths within the nose or sinuses characterized by trouble breathing through the nose, loss of smell, decreased taste, post-nasal drip, and a runny nose. Possibly $60-65 \%$ of the population suffering from CRS have predisposition to nasal polyp. ${ }^{1}$

Incidence of nasal polyp with the symptoms of CRS is seen to be on the rise in correlation to the increasing levels of environmental pollution and stress. ${ }^{2}$ CRS is a common disorder, with up to 35 million people being affected annually, and results in over 12 million office visits yearly. ${ }^{3}$ The physical symptoms of CRS are
1 - Department of Community Medicine, Bengal
Homoeopathic Medical College \& Hospital, Asansol, West Bengal
2 - Department of Repertory, National Institute of
Homoeopathy, Kolkata, West Bengal
3 - Independent Researcher, Central Council of
Homoeopathy, Howrah, West Bengal
Corresponding author:
Dr Aniruddha Banerjee
email: draniruddhabanerjee@gmail.com 
associated with functional and emotional impairment, which can lead to an overall poor quality of life (QOL). ${ }^{4,5}$ Likewise, obstructive sleep apnoea (OSA) is just as common. With up to 40 million Americans affected, it is increasing in prevalence and has been demonstrated in approximately $24 \%$ of men and $9 \%$ of women. Similar to CRS, it is associated with a poor QOL and functional and emotional impairment. ${ }^{6,7} \mathrm{CRS}$ is a common health condition in industrial countries concerning $10-15 \%$ of the German population. ${ }^{8}$

In the US, it was the most frequently reported chronic disease in a representative cohort of 100,000 adults participating in the National Health Interview Survey 1988. ${ }^{9}$ Negative effects of CRS on QOL of the patients have frequently been underestimated. Nevertheless, many patients report higher impacts of CRS on QOL dimensions like bodily pain or social functioning compared with other diseases like angina pectoris, pulmonary emphysema or chronic bronchitis. ${ }^{10}$ Economic impact of CRS has rarely been investigated. In 2006, nearly 45,000 patients with CRS were operated in German hospitals. ${ }^{11}$ In 1996, the healthcare expenditures for sinusitis in the US were 5.8 billion dollars. ${ }^{12}$ Gliklich and Metson ${ }^{13}$ performed a breakeven analysis and calculated a time period of 7 years until amortization of treatment costs can be achieved by reduced postoperative use of resources. Under these conditions, effective and successful treatment of NPs with CRS has an increasing medical and economic importance. In 1942, the revision surgery rate in 190 patients after trans-facial extra-nasal procedures was nearly 31\%.14 Modern Functional Endo-nasal Sinus Surgeries (FESS) resulted in stable subjective success rates between $70 \%$ and $92 \%{ }^{15,16,17}$

\section{Materials and Methods}

Trial setting and design: Samples were collected from amongst the patients attending the ENT out-patients. The study protocol was approved by the Institutional Ethical Committee (IEC). The trial protocol (unpublished) and full dissertation was submitted as the postgraduate thesis of the corresponding author to the West Bengal University of Health Sciences.
Inclusion criteria: All diagnosed cases of nasal polyp having symptoms of CRS, age 18 to 65 years, both sexes, patients willing to take part in the study and providing written consent. Patients availing any form of treatment for the same were enrolled after a wash-out phase of 1 month provided they had persistence of relevant signs and symptoms.

Exclusion criteria: Patients opting for surgical intervention, patients receiving active treatment from any other system of medicine, uncontrolled systemic or psychiatric illness, and patients denying consent.

Intervention: All medicines and vehicles in this project were procured from Homeopathy International - a GMP certified firm. All the patients were treated with individualized homoeopathic medicines in 50 millesimal potencies as per need of the case. In 50 millesimal potencies, a single medicated cane sugar globule of poppy seed size (no. 10) was dissolved in 80 $\mathrm{ml}$ distilled water with addition of 2 drops of $90 \% \mathrm{v} / \mathrm{v}$ ethanol, 16 doses marked on the vial, each dose of $5 \mathrm{ml}$ was instructed to be taken after 10 uniformly forceful downward strokes to the vial in $45 \mathrm{ml}$ normal water in a clean cup, to stir well, to take $5 \mathrm{ml}$ of this liquid orally, and to discard rest of the liquid from the cup.

Repetition was advised daily or every alternate day depending upon the individual requirements of the case and as decided appropriate by the treating homeopaths. Duration of therapy was 3 months. Single individualized medicine was prescribed on each occasion taking into account presenting symptoms totality, clinical history details, constitutional features, miasmatic expressions, repertorisation using the software RADAR $^{\circledR}$ and consensus between two homoeopaths. Dose was also individualized and was based on the homoeopaths' judgment of susceptibility and consensus. Subsequent prescriptions were generated as per Kent's observations and Hering's law. Participants were assessed similarly by the two homoeopaths in follow-up visits. One of the prescribers possessed a masters degree in homoeopathy with more than 30 years of experience of practicing classical homoeopathy and the corresponding author was a postgraduate trainee at NIH with 3 years of experience. All the homoeopaths involved were affiliated with state councils. 
Outcomes: Primary - translated Bengali version of the Sino-Nasal Outcome Test (SNOT) questionnaire; secondary-EQ-5D-5L questionnaire; available and prevalidated Bengali version [User license no. 24882]. The Sino-Nasal Outcome Test 20 (SNOT - 20) was one of the most widely used quality-of-life instruments for sinonasal conditions ${ }^{18-21}$ and was intended for populations of people with rhino-sinusitis, rather than simply rhinitis. The SNOT-20 was a self-administered multiple choice 20 items test that is usually scored with a single summary score (0-5) without domains or subscales. The items were assigned to five subgroups (nasal symptoms, para-nasal symptoms, sleep-related symptoms, social impairment and emotional impairment). The score of the SNOT-20 was calculated by summation of all the symptoms scores. Therefore, scale values of the SNOT-20 ranged from 0 to 100 . Additionally, patients could circle those 5 symptoms which have the highest impact on the impairment. This instrument assessed a broad range of health and health- related quality-oflife problem including physical problems, functional limitations and emotional consequence, but unlike many of the quality-of-life instruments designed to measure rhinitis symptoms, this rhino-sinusitis measure was not divided into subscales or domains related to these different areas. Another was the EQ-5D-5L questionnaire.

It was a standardized measure of health status developed by the Euro QoL Group ${ }^{22}$ in order to provide a simple, generic measure of health for clinical and economic appraisal. It was applicable to a wide range of health conditions and treatments; it provided a simple descriptive profile and a single index value for health status that could be used in the clinical and economic evaluation of healthcare as well as in population health surveys. EQ-5D was available in 139 languages including Bengali, was designed for self-completion by respondents and was ideally suited for use in postal surveys, in clinics, and in face-to-face interviews. It was cognitively undemanding, taking only a few minutes to complete. Instructions to respondents were included in the questionnaire..$^{23,24}$

Sample size: No relevant data was available on reduction of SNOT score by individualized homoeopathic treatment using an open observational study design over
3 months of intervention. Thus, assuming a medium effect size (d) of 0.5 , alpha $=0.05$ and power of $90 \%$, to detect a significant difference between two dependent means (mean pre- and post- SNOT scores) by paired $t$ test, we would have required a sample size of 44 .

Statistical methods: Intention to treat sample was subjected to statistical analysis. Missing values were replaced by Last value Carried Forward (LVCF) method. Data distribution was examined by histograms, Q-Q plots, Kolmogorov-Smirov and Shapiro-Wilk tests. Descriptive statistics was used to represent baseline data. Inferential statistics was used in terms of non-parametric Wilcoxon signed rank test and parametric post hoc paired $t$ test to compare SNOT-20 and EQ-5D-5L scores those obtained at baseline and after 3 months. $\mathrm{P}$ values less than 0.05 were considered as statistically significant. No interim and subgroup analyses were planned. SPSS $\AA$ IBM ${ }^{\circledR}$ version 20 software for Windows was used for analysis of data. Reporting adhered to the CONSORT ${ }^{25}$ and $\mathrm{RedHot}^{26}$ guidelines for reporting trials, Mathie's criteria for model validity of homoeopathic treatment ${ }^{27,28}$ (MVHT), and Saha's criteria for reporting quality of homoeopathic individualization in clinical trials. ${ }^{29}$

\section{Results}

Participant flow: As per the pre-specified inclusion and exclusion criteria, 70 patients suffering from nasal polyposis were screened; 26 were excluded on account of various reasons; 44 met the eligibility criteria and were enrolled into the study. Following that, baseline socio-demographic and outcome data were obtained $(n=40)$. After 3 months of intervention, outcome data was recorded again. During course of treatment, 4 dropped out; 40 completed the trial; 44 entered into the analysis. (Fig. 1)

Recruitment: Starting from May 2017 until November 2017 , total 44 participants were enrolled in the study.

Baseline data: Twelve socio-demographic features were studied - age (as continuous variable), age groups (categorical variable), gender, residence, duration of suffering, treatment taken, body mass index (BMI; as continuous variable), BMI classes (categorical variable), marital status, education, employment status, 


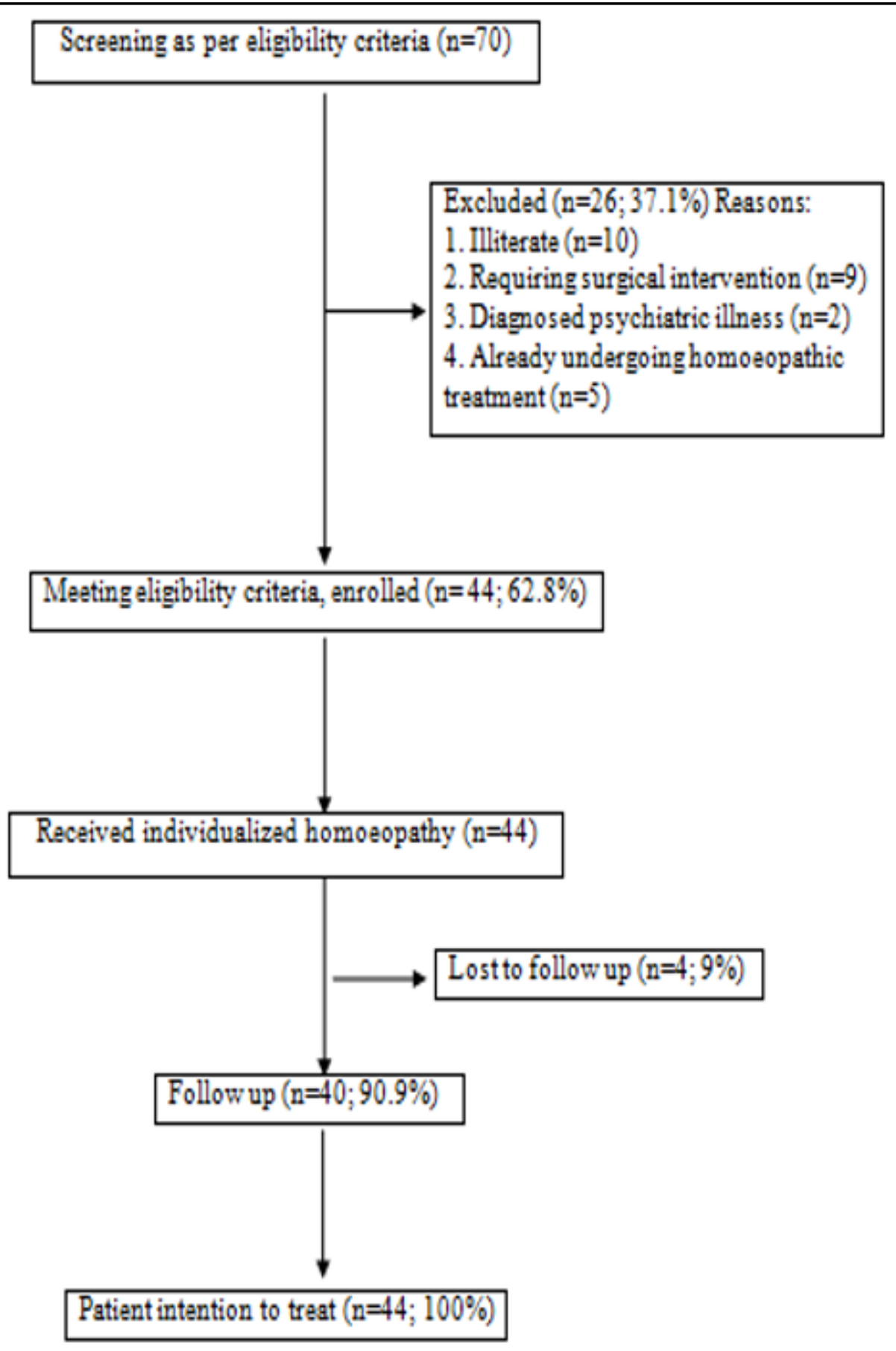

Fig. 1. Study flow diagram 
and family income status (Table 1)

Data distribution: It was examined by histograms (Supplementary Data - Figures: 1-6), Q - Q plots (Supplementary Data - Figures: 7-12) and statistical tests - Kolmogorov Smirnov test and Shapiro Wilk test (Supplementary Data - Table 1). Data distribution seemed to follow a mixed pattern - at baseline normal but non-normal after 3 months. Thus, first non-parametric Wilcoxon signed rank test (Table II) was planned to perform; and if significance was detected, then post hoc parametric paired t test (Table III).

Numbers analyzed: All the enrolled patients $(\mathrm{N}=44)$ entered into the final analyses.

\section{Changes in outcomes:}

1. As per skiagrams of 40 protocol-compliant cases, there were complete regressions of nasal polyps in $23(58 \%)$ cases, partial improvement in $11(27 \%)$ cases and no changes in $6(15 \%)$ cases.

2. After 3 months of treatment, there were statistically significant improvements in SNOT-20 scores, EQ-5D-5L questionnaire scores, and EQ-5D-5L VAS measures (all $\mathrm{P}<0.05$ ). (Tables II and III, Fig. 2)

Correlation statistics: The correlation scatter plots (Figure: 15 and 16) revealed weak to moderate positive linear relationships between changes in SNOT score and EQ-5D-5L scores.

Medicines used: The following medicines were prescribed - Tuberculinum $[\mathrm{n}=2 ; 5 \%]$, Thuja occidentalis $[n=1 ; 2.5 \%]$, Sulphur $[n=2 ; 5 \%]$, Sangunaria canadensis $[n=1 ; 2.5 \%]$ Pulsatilla nigricans $[n=2 ; 5 \%]$, Psorinum $[n=11 ; 27.5 \%]$, Nitric acid $[n=2 ; 5 \%]$, Natrum muriaticum $[n=1 ; 2.5 \%]$, Hepar sulphuricum $[n=3$; $7.5 \%]$, Dulcamara $[\mathrm{n}=10 ; 25 \%]$ Calcarea carbonica $[\mathrm{n}=5 ; 12.5 \%]$, Belladonna $[\mathrm{n}=1 ; 2.5 \%]$, Arnica montana $[\mathrm{n}=1 ; 2.5 \%]$. (Table IV)

Adverse events: No harm, unintended effects, serious adverse events, and homeopathic aggravations were reported during the study period.

\section{Discussion}

This prospective, open, non-randomized, single arm, observational clinical study of pre-post comparison

Table I: Baseline features

\begin{tabular}{|c|c|}
\hline Age $\Delta(y r s ;$ mean $\pm s d)$ & $30.0 \pm 10.5$ \\
\hline \multicolumn{2}{|l|}{ Age groups (yrs) $\S$} \\
\hline$\S 18-25$ & $20(45.5)$ \\
\hline$\S 26-40$ & $18(40.9)$ \\
\hline$\S$ Above 40 & $6(13.6)$ \\
\hline \multicolumn{2}{|l|}{ Gender $\S$} \\
\hline$\S$ Male : Female & $30(68.2): 14(31.8)$ \\
\hline \multicolumn{2}{|l|}{ Residence $§$} \\
\hline$\S$ Rural : Urban & $26(59.1): 18(40.9)$ \\
\hline $\begin{array}{l}\text { Duration of suffering } \Delta \\
\text { (yrs; mean } \pm \mathrm{sd})\end{array}$ & $3.5 \pm 2.3$ \\
\hline \multicolumn{2}{|l|}{ Treatment taken $\S$} \\
\hline § Allopathy & $11(25)$ \\
\hline § Homoeopathy & $11(25)$ \\
\hline$\S$ None & $22(50)$ \\
\hline BMI $\Delta($ mean \pm sd $)$ & $23.5 \pm 3.9$ \\
\hline \multicolumn{2}{|l|}{ BMI classes $\S$} \\
\hline § Underweight to normal & $26(59.1)$ \\
\hline$\S$ Overweight & $15(34.1)$ \\
\hline$\S$ Obese & $3(6.8)$ \\
\hline \multicolumn{2}{|l|}{ Marital status $\S$} \\
\hline$\S$ Single : Married & $19(43.2): 25(56.8)$ \\
\hline \multicolumn{2}{|l|}{ Educational status $\S$} \\
\hline$\S$ 8th std or below & $25(56.8)$ \\
\hline$\S 9$ th to 12 th std & $4(9.1)$ \\
\hline$\S$ Graduate or above & $15(34.1)$ \\
\hline \multicolumn{2}{|l|}{ Employment status $\S$} \\
\hline § Unemployed & $14(31.8)$ \\
\hline$\S$ Farmer & $5(11.4)$ \\
\hline$\S$ Business & $19(43.2)$ \\
\hline
\end{tabular}


Table I: Baseline features (contd.)

\begin{tabular}{|l|l|}
\hline Family income status $\S$ & \\
\hline § Low & $25(56.8)$ \\
\hline § Medium to affluent & $19(43.2)$ \\
\hline
\end{tabular}

$\triangle$ Continuous data presented as mean \pm standard deviations; $\S$ Categorical data presented as absolute values and percentages

design was conducted on 44 patients suffering from nasal polyps associated with CRS. SNOT-20 and EQ-5D-5L questionnaires were taken as the primary and secondary outcome measures respectively; assessed at baseline and after 3 months. Individualized homoeopathic medicines were prescribed on 'totality of symptoms.' Complete regression of polyps was achieved in $23(58 \%)$ cases. Statistically significant improvements were observed in both the outcomes over a period of 3 months. Thirteen individualized homoeopathic medicines were prescribed. Out of those, Psorinum [ $n=11 ; 27.5 \%]$ was the most frequently prescribed medicine. Individualized homoeopathic medicines seemed to be promising treatment for symptomatic nasal polyps.

The methodological strengths of the study included the prospective patient enrolment and use of standardized and already validated outcome scales SNOT-20 and EQ-5D-5L questionnaires. This study is representative of individualized homoeopathy only. In a broader interpretation of the law of similar, medicines were selected on the basis of 'totality of symptoms.' Observational trials are important for establishing hypothesis and the sample size for significant result. Though the sample size of this study was small, still was adequately powered to detect changes in the specified outcome measure over 3 months. The sample size had to be kept small keeping in mind the feasibility issues and stipulated time frame of postgraduate thesis. All the collected data (hard form) was converted into an analyzable and reproducible master chart (soft copy) where all data was extracted systematically and underwent statistical analysis subsequently.

At the end of the study, forty protocol-compliant

Table II: Changes in outcomes over time: analysis by Wilcoxon signed rank test $(\mathrm{N}=44)$

\begin{tabular}{|c|c|c|c|c|}
\hline \multirow{2}{*}{ OUTCOMES } & BASELINE & AFTER 3 MONTHS & \multirow{2}{*}{ Z SCORE } & \multirow{2}{*}{ P VALUE } \\
\hline & MEDIAN (IQR) & MEDIAN (IQR) & & \\
\hline SNOT-20 score & $33.5(25.5$ to 47.0$)$ & $16.5(13.3$ to 22.0$)$ & -5.472 & $<0.001$ \\
\hline \multicolumn{5}{|l|}{ EQ-5D-5L: } \\
\hline § Questionnaire score & $5.0(3.0$ to 6.0$)$ & $3.0(2.0$ to 5.8$)$ & -2.844 & 0.004 \\
\hline$\S$ VAS score & $60.0(50.0$ to 70.0$)$ & $74.0(62.0$ to 81.8$)$ & -5.528 & $<0.001$ \\
\hline
\end{tabular}

Table III: Changes in outcomes over time: pair-wise analysis by post hoc paired $t$ test $(\mathrm{N}=44)$

\begin{tabular}{|c|c|c|c|}
\hline \multirow{2}{*}{ OUTCOMES } & BASELINE; & AFTER 3 MONTHS; & CHANGE (0-3 MO); \\
\cline { 2 - 4 } & MEAN \pm SD & MEAN \pm SD & MEAN \pm SD (95\% CI) \\
\hline SNOT-20 score & $37.0 \pm 16.3$ & $18.5 \pm 7.7$ & $18.5 \pm 14.2(14.2$ to 22.9$) \S$ \\
\hline EQ-5D-5L: & & & \\
\hline \& Questionnaire score & $4.6 \pm 2.5$ & $3.6 \pm 2.6$ & $1.0 \pm 2.4(0.3$ to 1.8$) \psi$ \\
\hline$\S$ VAS score & $\mathbf{5 8 . 0} \pm 14.0$ & $\mathbf{7 2 . 8} \pm 12.5$ & $14.8 \pm 9.6(11.9$ to 17.7$) *$ \\
\hline
\end{tabular}

$\S t 43=8.660, P<0.001$ (significant) $; \psi t 43=2.831, P=0.007$ (significant) $* t 43=10.211, P<0.001$ (significant) 


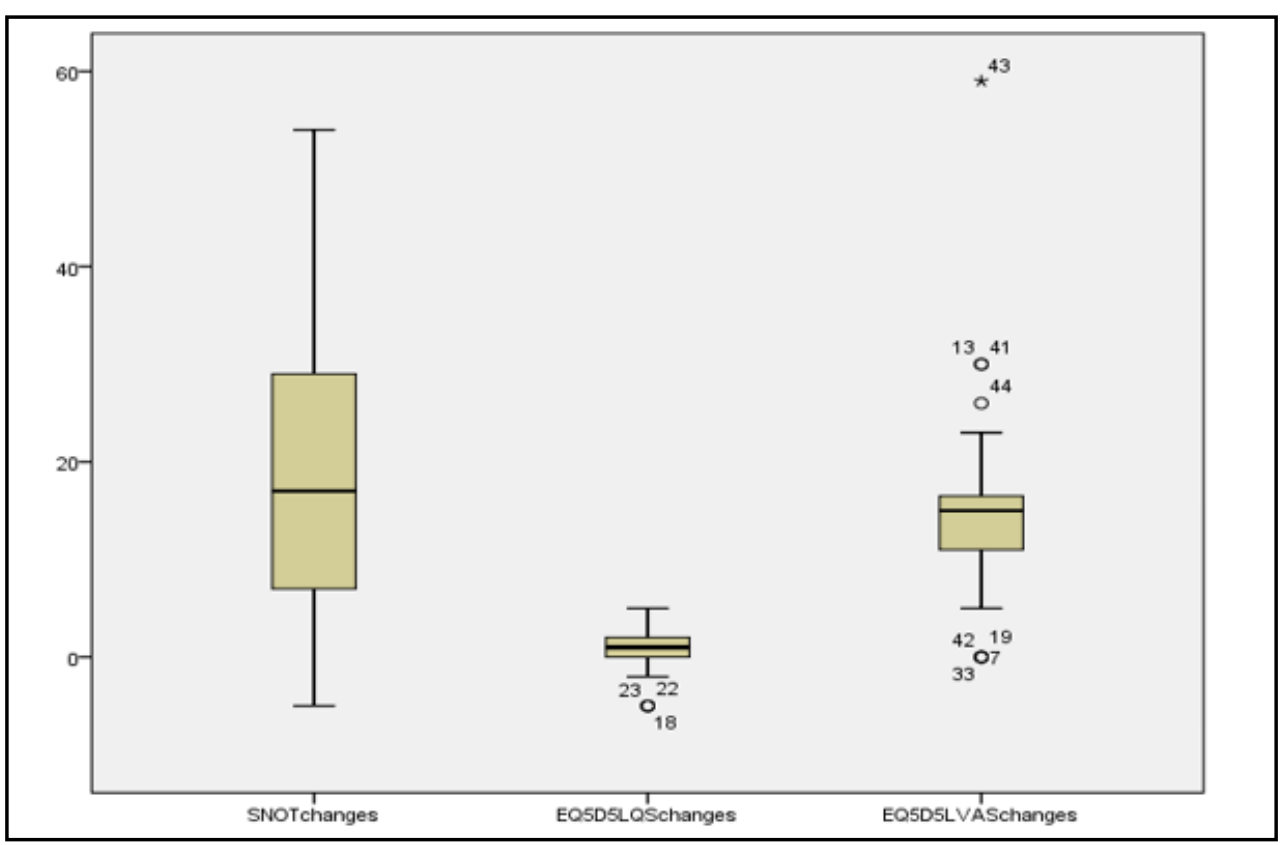

Fig. 2. Box and whisker plots showing changes in SNOT-20 scores, EQ-5D-5L questionnaire scores and EQ-5D-5L VAS scores over 3 months of treatment $(\mathrm{N}=44)$; SNOT-20 changes: Median 17.0, IQR 7.0 to 29.0; EQ-5D-5L questionnaire score changes: Median 1.0, IQR 0.0 to 2.0; EQ-5D-5L VAS score changes: Median 15.0, IQR 11.0 to 16.8

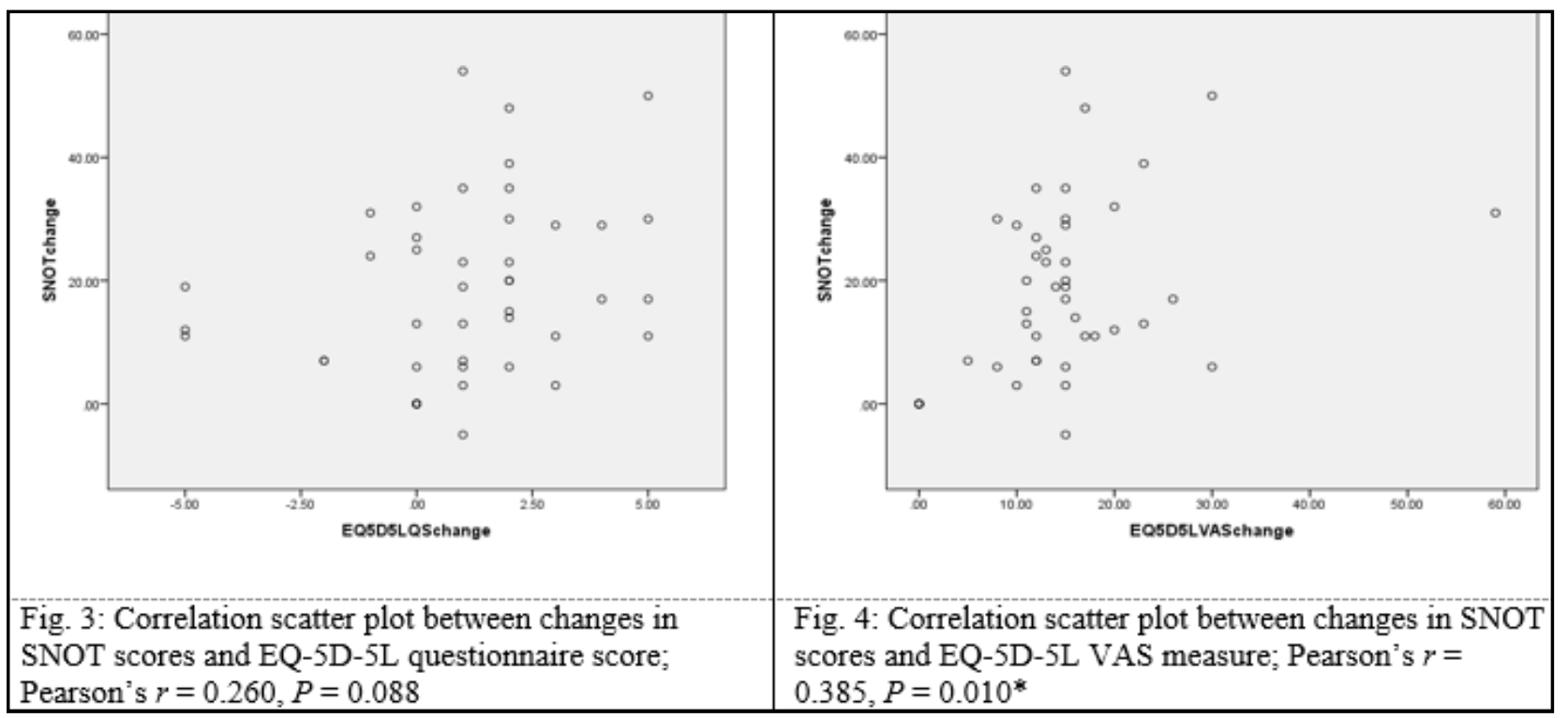

Figs. 3 and 4. Correlation statistics between changes in outcomes 
Table IV: Used medicines

\begin{tabular}{|c|c|c|}
\hline $\begin{array}{c}\text { PRESCRIBED } \\
\text { MEDICINES }\end{array}$ & $\begin{array}{c}\text { NO. OF } \\
\text { PRESCRIPTIONS }\end{array}$ & PERCENTAGES (\%) \\
\hline Tuberculinum & 2 & 5 \\
\hline Thuja occidentalis & 1 & 2.5 \\
\hline Sulphur & 2 & 2.5 \\
\hline Sangunaria canadensis & 1 & 5 \\
\hline Pulsatilla nigricans & 2 & 27.5 \\
\hline Psorinum & 11 & 5 \\
\hline Nitric acid & 2 & 2.5 \\
\hline Natrum muriaticum & 1 & 7.5 \\
\hline Hepar sulphuris & 3 & 25 \\
\hline Dulcamara & 10 & 12.5 \\
\hline Calcarea carbonica & 5 & 2.5 \\
\hline Belladonna & 1 & 2.5 \\
\hline Arnica montana & 1 & \\
\hline
\end{tabular}

patients entered into the final analysis. Descriptive statistics was used to represent baseline data. Inferential statistics was used in terms of paired $t$ test. Statistically significant improvements were observed in both the outcomes - SNOT-20 scores and EQ5D-5L questionnaire scores. Statistically significant improvements were observed in both the outcomes. There was no violation of routine homoeopathy practice and the outcome data was gathered at baseline and 3 months of treatment. All the medicines were prescribed in fifty millesimal potencies. Homoeopathic medicines provided promising aid in treatment of symptomatic nasal polyps.

Constraints in sample size and duration of followup were the major limitations of the study. This study did not support conclusions as to the efficacy or effectiveness of the homoeopathic medicines because no methodology for this purpose (control group, randomization, blinding, etc.) was built into its design. Another limitation was inherent in the observational design of the study, i.e. the trial was open; no blinding was used. Thus, patient selection bias, evaluation bias, and data analysis bias might have affected the outcomes of the study. No causal inference could be attributed to the treatment and the treatment could not be regarded as the sole reason for the changes in the patients. Also, the other limitation was to use the subjective measures, namely SNOT-20 and EQ-5D-5L questionnaires, that might have incorporated some kind of bias.

Validity and reliability of the translated Bengali version of SNOT-20 questionnaire remain to be addressed formally in future studies. The data may also be helpful in the planning of further controlled research in homoeopathy. It would require specific objective measures for valid assessment and a longer observation period. Further independent replications and explorations by randomized trials with enhanced methodological rigor are warranted.

\section{Conclusion}

In this prospective, open, non-randomized, single arm, observational clinical study of pre-post comparison design conducted on 44 patients suffering 
from symptomatic nasal polyps revealed complete regressions of polyps in $23(58 \%)$ cases and statistically significant improvement in both SNOT-20 and EQ5D-5L questionnaires after 3 months of individualized homoeopathic treatment. Further explorations by randomized trials are warranted.

\section{References}

1. Murphy MP, Fishman P, Short SO, Sullivan SD, Yueh B, Weymuller EA Jr. Health care utilization and cost among adults with chronic rhinosinusitis enrolled in a health maintenance organization. Otolaryngol Head Neck Surg. 2002; 127:367-76

2. Anand VK: Epidemiology and economic impact of rhinosinusitis. Ann Otol Rhinol Laryngol Suppl. 2004; 193:3-5

3. Jackson LL, Kountakis SE. Classification and management of rhinosinusitis and its complications. Otolaryngol Clin North Am. 2005; 38:1143-53

4. Smith TL, Batra PS, Seiden AM, Hannley M. Evidence supporting endoscopic sinus surgery in the management of adult chronic rhinosinusitis: a systematic review. Am J Rhinol. 2005; 19:537-43

5. Gliklich RE, Metson R. The health impact of chronic sinusitis in patients seeking otolaryngologic care. Otolaryngol Head Neck Surg. 1995; 113:104-9

6. Piccirillo JF. Outcomes research and obstructive sleep apnea. Laryngoscope 2000;110:16-20

7. National Commission on Sleep Disorders Research. Wake Up America: A National Sleep Alert. Washington, Government Printing Office, 1993

8. Pade J. Sinusitis. Eine ernst zu nehmende Erkrankung. HNO 2005; DOI 10.1007/s00106-005-1227-0

9. Pleis JR, Coles R. Summary health statistics for US adults: Nation Health Interview Survey, 1998. National Center for Health Statistics. Vital Health Stat 2002; 10: 1-113

10. Becker DG. Sinusitis. J Long Term Eff Med Implants. 2003; 13:175-94

11. Gesundheitsberichterstattung des Bundes. Available from: http://www.gbe-bund.de/ Accessed on October 13, 2017

12. Ray NF, Baraniuk JN, Thamer M, et al. Healthcare expenditures for sinusitis in 1996: contributions of asthma, rhinitis, and other airway disorders. J Allergy Clin Immunol. 1999; 103: 408-14

13. Metson R, Gliklich RE. Clinical outcome of endoscopic surgery for frontal sinusitis. Arch Otolaryngol Head Neck Surg. 1998; 124:1090-6

14. Goodale RL. Some causes of failure in frontal sinus surgery. Ann Otol. 1942; 51:648
15. Iro H, Mayr S, Wällisch C, Schick B, Wigand ME. Endoscopic sinus surgery: its subjective medium-term outcome in chronic rhinosinusitis. Rhinology. 2004; 42:200-6

16. Sobol SE, Wright ED, Frenkiel S. One-year outcome analysis of functional endoscopic sinus surgery for chronic sinusitis. J Otolaryngol. 1998; 27:252-7

17. Stammberger H, Posawetz G. Functional endoscopic sinus surgery. Eur Arch Otorhinolarngol. 1990; 247:63-76

18. Hessler JL, Piccinllo JF, Fang D, et al. Clinical outcomes of chronic rhinosinusitis in response to medical therapy: Results of prospective study. Am J Rhinol. 2007; 21:10-8

19. Robinson S, Douglas R, and wormald PJ. The relationship between atopy and chronic rhinosinusitis. Am J Rhinol. 2006; 20:625-8

20. Piccinllo JF, Merritt MG Jr, Richards ML. Psychometric and clinimetric validity of the 20- Item Sino-Nasal Outcome Test(SNOT-20). Otolaryngol Head Neck Surg. 2002; 126:41-7

21. Browne JP, Hopkins C, Slack R, Cano SJ. The Sino-Nasal Outcome Test (SNOT): can we make it more clinically meaningful? Otolaryngol Head Neck Surg. 2007; 136:736-41

22. The Euro-QOL group. EQ-5D-5L 2015. Available from: http:// www.euroqol.org/eq- 5d-products/eq-5d-51.html; last accessed July 18, 2015

23. The EuroQol Group. EuroQol - a new facility for the measurement of health-related quality of life. Health Policy. 1990;16 (3):199-208

24. Herdman M, Gudex C, Lloyd A, Janssen MF, Kind P, Parkin $\mathrm{D}$, et al. Development and preliminary testing of the new fivelevel version of EQ-5D (EQ-5D-5L). Qual Life Res. 2011;20 (10):1727-36

25. Schulz KF, Altman DG, Moher D, for the CONSORT group. CONSORT 2010 statement: updated guidelines for reporting parallel group randomized trials. BMJ 2010; 340:c332

26. Dean ME, Coulter MK, Fisher P, Jobst K, Walach H. Reporting data on homoeopathic treatments (RedHot): a supplement to CONSORT. Homoeopathy 2007: 96(1):42-5

27. Mathie RT, Van Wassenhoven M, Jacobs J, Oberbaum M, Roniger H, Frye J, et al. Model validity of randomized placebocontrolled trials of individualized homoeopathic treatment. Homoeopathy 2017; 106 (4):194-202

28. Mathie RT, Van Wassenhoven M, Jacobs J, Oberbaum M, Frye J, Manchanda RK et al. Model validity and risk of bias in randomized placebo-controlled trials of individualized homoeopathic treatment. Complement Ther Med. 2016; 25:120-5

29. Saha S, Koley M, Ganguly S, Rath P, Roy Chowdhury P, Hossain SI. Developing the criteria for evaluating quality of individualization in homoeopathic clinical trial reporting a preliminary study. J Integr Med. 2014; 12(1):13-9. 